\title{
artigo
}

Marçal, T.V.G.; Costa, L.F.; Nicoletti, D.R.; Fernandes, M.T.C.; Amorin, B.; Hermes, D.;

Incidência de KPC (Klebsiella Pneumoniae Carbapenemase) em adultos internados em hospitais nas regiões do Brasil de 2006 a 2016 : revisão bibliográfica

\section{Incidência de KPC (Klebsiella Pneumoniae Carbapenemase) em adultos internados em hospitais nas regiões do Brasill de 2006 a 2016: revisão bibliográfica}

Incidencia de KPC (Klebsiella Pneumoniae Carbapenemase) en adultos ingresados en hospitales de las regiones de Brasil de 2006 a 2016: revisión de la literatura

Incidência de KPC (Klebsiella Pneumoniae Carbapenemase) em adultos internados em hospitais nas regiões do Brasil de 2006 a 2016: revisão bibliográfica

\begin{abstract}
RESUMO
A Klebsiella pneumoniae carbapenemase (KPC), conhecida como "superbactéria", desempenha um papel importante em relação às infecções hospitalares. Objetivos: Identificar a incidência de Klebsiella pneumoniae com fator de resistência KPC em adultos nos hospitais das regiões Centro-Oeste, Sudeste e Sul do Brasil entre os anos de 2006 e 2016, observando também, o perfil de resistência aos antimicrobianos. Método: Pesquisa retrospectiva com base descritiva e qualitativa, sendo uma revisão bibliográfica literária sistemática realizada por meio das bases de dados: SciELO, Medline, LILACS e Pubmed. Resultados: Localizou-se 95 artigos, dos quais 58 foram incluídos. A região Sul obteve a maior prevalência para isolados KPC nos hospitais, sendo a Klebsiella pneumoniae. Em relação aos antimicrobianos o Ertapenem teve quase $100 \%$ de resistência em todos os estados. Conclusão: $\bar{E}$ necessário que sejam implementadas precauções e o controle da disseminação desse tipo de mecanismo de resistência.
\end{abstract}

DESCRITORES: Resistência bacteriana; Enterobactérias; Epidemiologia.

\begin{abstract}
Klebsiella pneumoniae carbapenemase (KPC), known as "superbug", plays an important role in relation to nosocomial infections. Objectives: To identify the incidence of Klebsiella pneumoniae with resistance factor KPC in adults in hospitals in the Midwest, Southeast and South regions of Brazil between the years 2006 and 2016, also observing the profile of resistance to antimicrobials. Method: Retrospective research with descriptive and qualitative basis, being a systematic literary literature review carried out through the databases: SciELO, Medline, LILACS and Pubmed. Results: 95 articles were found, of which 58 were included. The South region obtained the highest prevalence for KPC isolates in hospitals, being Klebsiella pneumoniae. Regarding antimicrobials, Ertapenem had almost 100\% resistance in all states. Conclusion: It is necessary to implement precautions and control the spread of this type of resistance mechanism.
\end{abstract}

DESCRIPTORS: Bacterial resistance; Enterobacteria; Epidemiology.

\section{RESUMEN}

Klebsiella pneumoniae carbapenemase (KPC), conocida como "superbacteria", juega un papel importante en relación con las infecciones nosocomiales. Objetivos: Identificar la incidencia de Klebsiella pneumoniae con factor de resistencia KPC en adultos en hospitales de las regiones Medio Oeste, Sudeste y Sur de Brasil entre los años 2006 y 2016, observando también el perfil de resistencia a los antimicrobianos. Método: Investigación retrospectiva con base descriptiva y cualitativa, siendo una revisión sistemática de la literatura literaria realizada a través de las bases de datos: SciELO, Medline, LILACS y Pubmed. Resultados: se encontraron 95 artículos, de los cuales se incluyeron 58. La región Sur obtuvo la mayor prevalencia de aislamientos de KPC en hospitales, siendo Klebsiella pneumoniae. Con respecto a los antimicrobianos, Ertapenem tenía casi el 100\% de resistencia en todos los estados. Conclusión: Es necesario implementar precauciones y controlar la propagación de este tipo de mecanismo de resistencia.

DESCRIPTORES: Resistencia bacteriana; Enterobacterias; Epidemiología.

RECEBIDO EM: 13/12/2020 APROVADO EM: 23/12/2020

Tássia Vieira Goularte Marçal

Bacharel em Biomedicina, Centro Universitário Ritter dos Reis (UniRitter).

ORCID: 0000-0002-1504-8790 


\section{Letícia Falkenbach da Costa}

Residente multiprofissional em saúde comunitária - ULBRA, Canoas - RS, Brasil.

ORCID: 0000-0003-1817-6971

\section{Diana Rodrigues Nicoletti}

MBA em Gestão em Saúde e Controle de Infecção, Faculdade CEAT.

ORCID: 0000-0003-3393-0362

\section{Morgana Thais Carollo Fernandes}

Pós- Doutoranda em Saúde da Criança pela Pontifícia Universidade Católica do Rio Grande do Sul (PUCRS), bolsista pela University of Toronto (UofT). Professora Colaboradora da Residência Multiprofissional em Saúde da Criança e Pesquisadora Associada do Programa de Extensão e Pesquisa em Saúde Urbana, Ambiente e Desigualdades da Universidade Federal do Rio Grande do Sul (UFRGS). - Porto Alegre, RS.

ORCID: 0000-0002-7989-294X

\section{Bruna Amorin}

Pós Doutorado em Medicina - UFRGS. Docente da Faculdade Inedi - Cesuca - Cachoeirinha, RS, Brasil. ORCID: 0000-0002-8912-9171

\section{Djuli Hermes}

Mestre em Medicina - UFRGS. Docente da Insituição UniRitter - Porto Alegre - RS, Brasil. ORCID: 0000-0002-6466-1743

\section{INTRODUÇÃO}

A enzima KPC (Klebsiella pneumoniae carbapenemase), ficou conhecida como "superbactéria", após ter sofrido uma mutação genética, que lhe conferiu resistência a múltiplos antimicrobianos e a outras bactérias. Esta enzima foi identificada pela primeira vez nos Estados Unidos, em 1996, encontrada pela primeira vez na bactéria Klebsiella Pneumoniae, daí o nome recebido ${ }^{1}$.

Rapidamente a enzima disseminou-se por vários países, a Colômbia em 2006 foi o primeiro país Sul Americano a registrar a ocorrência de casos de KPC-2, em isolados de Klebsiella pneumoniae ${ }^{1-2}$. Apesar do primeiro registro brasileiro ocorrer em 2005, apenas em 2011 passou-se a observar surtos mais graves referentes a bactérias produtoras de KPC. O primeiro surto de infecção nosocomial ocorreu em 2009, mas só foi registrado em $2011^{1}$.

A KPC é uma enzima produzida por bactérias gram-negativas (enterobactérias), restritas ao ambiente hospitalar, que confere resistência aos antimicrobianos carbapenêmicos, amplamente utilizados no tratamento de infecções envolvendo Enterobacteriaceae, como: Meropenen, Ertapenen, Imipenen, além de inativar outros agentes Beta-lactâmicos: cefalosporinas, penicilinas e monobactâmicos ${ }^{3-7}$.

Em outras bactérias, como: Enterobacter cloacae, Citrobacter freundii, Salmonella spp., E. coli e Pseudomonas spp.8. A K. pneumoniae é uma bactéria com grande capacidade de transferir seu material genético, devido sua localização plasmidial e consequentemente, os genes de resistência ${ }^{9-11}$.

A fácil disseminação dificulta o controle de epidemias, e preocupa os profissionais da área de saúde, pois o tratamento destas infecções é extremamente difícil, elevando as taxas de mortalidade. Vários fatores estão envolvidos na disseminação desses patógenos multirresistentes, incluindo o uso abusivo de antibióticos, procedimentos invasivos, técnica inadequada ou ausência de higiene de mãos e a falta de rotina na desinfecção de superfícies ${ }^{8,12-13}$.

O uso de antibióticos ajudou na contenção das infecções e o seu sucesso gerou grande otimismo em relação à prevenção e ao tratamento dos processos infecciosos. Entretanto, a prática indiscriminada de antibioticoterapia trouxe o desenvolvimento de mecanismos de resistência comuns, resultando em uma queda contí- nua da eficácia da maioria dos antimicrobianos ao longo das últimas décadas ${ }^{8,14}$. Entre 2000 e 2010 o uso de antibióticos aumentou em 36\% em 71 países, Brasil, Rússia, Índia, China e África do Sul foram responsáveis por $76 \%$ desse aumento ${ }^{14}$.

A problemática em torno das infecções hospitalares ainda é um grande desafio à saúde pública em todo mundo ${ }^{8}$. Algumas publicações relatam taxas de mortalidade entre $40 \%$ a $50 \%$ para as enterobactérias produtoras de carbapenemases ${ }^{15}$. A prevenção é a arma principal no combate às bactérias produtora de carbapenemase, já que o tratamento é difícil devido sua alta resistência a antimicrobianos. Desta forma, todos os cuidados devem ser minuciosos quanto às infecções causadas por essas bactérias multirresistentes, pois representam um sério problema de saúde pública e um grande desafio terapêutico ${ }^{14,16-17}$.

Nesta perspectiva, considerando a importância do tema, o objetivo desse estudo foi realizar um levantamento da incidência epidemiológica dessas bactérias em hospitais das regiões Centro-Oeste, Sudeste e Sul do Brasil, através da revisão bibliográfica para observação de sua disseminação e da resistência aos antimicrobianos. 


\section{artigo}

Marçal, T.V.G.; Costa, L.F.; Nicoletti, D.R.; Fernandes, M.T.C.; Amorin, B.; Hermes, D.;

Incidência de KPC (Klebsiella Pneumoniae Carbapenemase) em adultos internados em hospitais nas regiões do Brasil de 2006 a 2016 : revisão bibliográfica

\section{MÉTODOS}

Estudo retrospectivo com base descritiva e qualitativa, realizado através das bases de dados: SciELO, Medline, LILACS e Pubmed sendo uma revisão bibliográfica literária sistemática nos idiomas Espanhol, Inglês e Português. O tema baseou-se em incidência de bactérias produtoras de KPC em ambientes hospitalares, em adultos, nas regiões Centro-Oeste, $\mathrm{Su}$ deste e Sul do Brasil nos anos de 2006 a 2016 para uma coleta de dados epidemiológicos e uma perspectiva da resistência bacteriana devido ao uso indiscriminado da antibióticopterapia.

Os dados foram analisados e tratados conforme o número de isolados KPC nas regiões e seus respectivos estados; bactéria com mais frequência, sendo está a Kblebsiella Pneumoniae Carbapenemase; gênero mais acometido; e fonte de isolamento com maior frequência. $\mathrm{O}$ número total de artigos somou-se 95, dos quais 37 foram excluídos e 58 incluídos para a realização do estudo. Os critérios para exclusão basearam-se em artigos dos quais não compreendiam o ano estabelecido; a idade dos pacientes; o fator de resistência bacteriana; as regiões pré-estabelecidas e o objetivo para levantamento epidemiológico. Foi critério para inclusão o público adulto; incidência de KPC em ambientes hospitalares entre os anos de 2006 e 2016; as regiões estabelecidas; com artigos mais atuais possíveis e dados de relevância para um levantamento epidemiológico regional e nacional.

Os dados epidemiológicos foram coletados de artigos das três regiões do Brasil, levando em consideração a prevalência de KPC por cada região do Brasil analisada e o número de isolados bacterianos positivos para Kblebsiella Pneumoniae Carbapenemase; prevalência e número da bactéria com maior frequência em isolados KPC; gênero; média de idade; fonte dos isolados; ano e a resistência dos antibióticos carbapenêmicos.

\section{RESULTADOS}

Cada região e seus estados foram analisadas separadamente, entre os anos de 2006 a 2016. Nas três regiões, o KPC prevaleceu a idade de 53 anos (mediana de 56,3). Houve predomínio do gênero masculino (51\%). Poucos estudos evidenciaram o público feminino (7\%) e alguns não especificavam o gênero (42\%), devido ao fato de não ser influência na susceptibilidade das infecções.

A fonte de isolamento das bactérias com fator KPC nas três regióes, teve predomínio para amostras de urina (35\%), seguido de swab retal (17\%), hemocultura (16\%), secreções traqueais (5\%), fragmentos de amputação (3\%) e cateter venoso (1\%). Destacou-se o elevado número de fontes não especificadas (23\%).

Entre todas as regiões, foram 6.184 isolados positivos para $\mathrm{KPC}$, sendo a $\mathrm{K}$. pneumoniae, a bactéria com maior frequência nesses isolados, compreendendo em 3.703 o número total (Tabela 1 ).

Tabela 1- Prevalência de isolados positivos para KPC em hospitais do Centro-Oeste, Sudeste e Sul do Brasil.

\begin{tabular}{|c|c|c|c|c|c|}
\hline Regiões Estados & $\begin{array}{l}\mathrm{N}^{\circ} \text { de isolados } \\
\mathrm{KPC}\end{array}$ & $\begin{array}{l}\text { Bactéria com maior } \\
\text { frequência }\end{array}$ & $\begin{array}{l}\text { Isolados com maior } \\
\text { frequência }\end{array}$ & Gênero & $\begin{array}{l}\text { Fonte de Isolamento com maior } \\
\text { prevalência }\end{array}$ \\
\hline \multicolumn{6}{|l|}{ Centro Oeste } \\
\hline Distrito Federal & 1.320 & K. pneumoniae & 770 & masculino & Urina \\
\hline Goiás & 160 & K. pneumoniae & 105 & masculino & Urina \\
\hline Mato Grosso & 4 & K. pneumoniae & 2 & masculino & Aspirado Traqueal \\
\hline TOTAL & 1.484 & K. pneumoniae & 877 & masculino & Urina \\
\hline \multicolumn{6}{|l|}{ Sudeste } \\
\hline Minas Gerais & 391 & K. pneumoniae & 123 & masculino & Hemocultura \\
\hline Espírito Santo & 37 & K. pneumoniae & 20 & masculino & Urina \\
\hline Rio de Janeiro & 276 & K. pneumoniae & 115 & masculino & Urina \\
\hline São Paulo & 156 & K. pneumoniae & 145 & masculino & Urina \\
\hline TOTAL & 860 & K. pneumoniae & 403 & masculino & Urina \\
\hline \multicolumn{6}{|l|}{ Sul } \\
\hline Rio Grande do Sul & 831 & K. pneumoniae & 433 & masculino & Urina \\
\hline Santa Catarina & 1.325 & K. pneumoniae & 1.210 & masculino & Urina \\
\hline Paraná & 1.644 & K. pneumoniae & 780 & masculino & Swab retal \\
\hline TOTAL & 3.840 & K. pneumoniae & 2.423 & masculino & Urina \\
\hline TOTAL REGIÕES & 6.184 & K. pneumoniae & 3.703 & masculino & Urina \\
\hline Fonte: Autor (2020). & & & & & \\
\hline
\end{tabular}


A bactéria $\mathrm{K}$. pneumoniae foi a mais frequente no estudo perfazendo $84 \%$, Acinetobacter baumannii (2\%), E. aerogenes (1\%) e E. cloacae (1\%), destacando-se também um número considerável de bactérias não especificadas para o fator $\mathrm{KPC}$ com $12 \%$.

Pode-se observar que no estado do Paos anos 2009 e 2015, houve um crescente aumento de isolados KPC, totalizando em 1.644 amostras. Nesta mesma região entre os anos 2006 e 2016, o estado de Santa Catarina (SC) apresentou elevada incidência para K. pneumoniae com fator de resistência para bactérias produtoras de KPC comparada aos demais estados. $\mathrm{Na}$ região Centro-Oeste entre os anos 2006 e 2013, especificamente no Distrito Federal (DF) houve acentuada taxa para o fator KPC, compreendendo 1.320 isolados. Em uma perspectiva geral, observou-se a prevalência de bactérias produtoras de KPC no Brasil, sendo a região Sul a de maior incidência seguido das regiões $\mathrm{Su}$ deste e Centro-Oeste, respectivamente, entre os anos 2006 e 2016 (Gráfico 1).

$\mathrm{Na}$ região Centro-Oeste, os estados de Distrito Federal (DF), Goiás (GO) e Mato Grosso (MT), 11 estudos reportaram menor prevalência para o número de raná (PR), na região Sul do Brasil, entre

isolados $\mathrm{KPC}$, comparado aos demais. $\mathrm{O}$ DF foi o estado com maior prevalência de números de isolados KPC em 1.320 de um total de 1.484 , sendo a K. pneumoniae a bactéria com maior frequência, com 770 isolados de 877 , resultando em $75 \%$. Destes, prevaleceu como fontes de isolamento urina (39\%), hemocultura (17\%), aspirado traqueal (6\%) swab retal (5\%) e não especificado (33\%), sendo o sexo masculino o mais acometido com $50 \%$, onde $44 \%$ não foram especificados e somente $6 \%$ eram do sexo feminino.

Na região Sudeste, 16 estudos foram investigados. Esta foi a região intermediária em relação à prevalência de isolados $\mathrm{KPC}$, quando comparada as demais. Os estados que fizeram parte desta pesquisa formam um total de quatro estados, sendo eles: Minas Gerais (MG), Espírito Santo (ES), Rio de Janeiro (RJ) e São Paulo (SP). Apresentou-se um total de 860 isolados, sendo a bactéria K. pneumoniae como a mais frequente, com um número de 303 isolados, resultando em 63\% das amostras.

$\mathrm{O}$ estado com maior número de isolados foi o MG, totalizando em 391 para o fator KPC e 123 com prevalência da K. pneumoniae. Do total de isolados dessa região, a maioria foi proveniente de fon-

\section{Gráfico 1- Levantamento epidemiológico conforme os estados selecionados, para incidência de fator KPC, K. pneumoniae e outras bactérias de 2006 a 2016.}

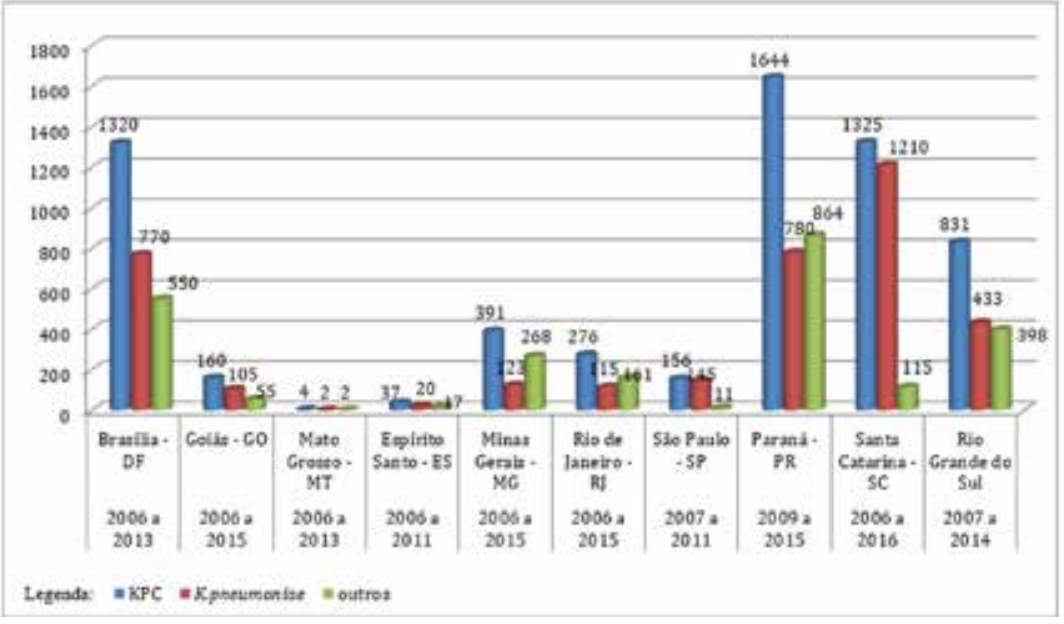

Fonte: Autor (2020) te de isolamento de urina (38\%), seguido de hemocultura (24\%), swab retal (14\%), secreções traqueais (3\%) e não especificado (24\%). O sexo masculino foi o mais acometido com $45 \%$ em relação ao gênero feminino, ainda $48 \%$ para gêneros não especificados.

Já a região Sul, foi a mais prevalente em termos de trabalhos analisados, totalizando 23 artigos. Esta região teve a maior prevalência para os isolados KPC, totalizando em 3.064 isolados positivos KPC com incidência de K. pneumoniae em 2.423 achados, dos quais resultaram em $92 \%$ das amostras. Os estados que fizeram parte dessa pesquisa foram: Rio Grande do Sul (RS), Santa Catarina (SC) e Paraná (PR).

$\mathrm{O} P R$ teve a maior prevalência com 1.644 isolados de KPC, sendo a K. pneumoniae também a bactéria mais comum nos achados, porém, foi no estado de SC a que a K. pneumoniae teve uma maior frequência, com um total de 1.210 isolados, mas o número de KPC total no estado foi menor do que o estado do PR. A fonte de maior isolamento foi de urina com $31 \%$, seguido de swab retal (27\%), não especificado $(18 \%)$, hemocultura (9\%), fragmento de amputação e secreções traqueais (6\%) e cateter venoso (3\%). O sexo masculino também foi o de maior prevalência com $58 \%$ dos achados.

Em relação aos antimicrobianos, foram selecionados 16 autores para o levantamento de resistência e sensibilidade dos isolados de bactérias com fator $\mathrm{KPC}$, cuja $K$. pneumoniae teve a maior prevalência em todas as regiões e estados em estudo.

Na região Centro-Oeste, entre os anos de 2006 a 2013, o Ertapenem teve maior prevalência como antimicrobiano com maior resistência em todos os estados, GO e MT com $100 \%$ e o DF com $92,8 \%$, seguido do Meropenem como o segundo com maior resistência, DF (92,5\%), GO (90\%) e MT (50\%). A menor resistência foi encontrada na Gentamicina com 57\% no DF e GO, sem prevalência para o MT. Já o antibiótico com maior sensibilidade foi a Amicacina no DF, com $96 \%$ e em GO com 92,2\%, também não houve prevalência para a média no $M T$. 


\section{artigo}

Marçal, T.V.G.; Costa, L.F.; Nicoletti, D.R.; Fernandes, M.T.C.; Amorin, B.; Hermes, D.;

Incidência de KPC (Klebsiella Pneumoniae Carbapenemase) em adultos internados em hospitais nas regiões do Brasil de 2006 a 2016 : revisão bibliográfica

Na região Sudeste, de 2006 a 2015, o Ertapenem teve 100\% de resistência em três estados, ES, MG e RJ, já em SP, não apresentou estudos para maior resistência. O Meropenem foi o segundo antibiótico com maior resistência, sendo o ES o estado com maior prevalência de $94,55 \%$. A Gentamicina ficou como o antibiótico de menor resistência em todos os estados, compreendendo em $12 \%$ em SP e $31 \%$ no ES. A maior média para sensibilidade também foi a Gentamicina com 95,6\% em MG, seguido da Amicacina com 95,6\% no RJ e $94,7 \%$ em SP.

Na região Sul, de 2006 a 2014, SC teve 100\% de resistência ao Ertapenem e o RS com 97,2\%. Porém no ano de 2011, no estado do PR, o Ertapenem também teve maior resistência, mas com apenas $40 \%$. O segundo com maior resistência foi o Meropenem, como nas outras regióes, compreendendo em 94,55\% em SC e $91,4 \%$ no RS, apresentando também uma baixa no estado do PR, com 32\%. A menor resistência foi da Gentamicina, com $28,2 \%$ no RS. A maior sensibilidade foi da Fosfomicina, com 100\% no RS, 92,2\% em SC e no estado do PR o Imipenem foi o resultado para maior sensibilidade, com 83\% (Tabela 2).

\section{DISCUSSÃO}

As IRAS (Infecções Relacionadas à Assistência à Saúde) causadas pelas bactérias Gram-negativas traz enorme preocupação em todo mundo, especialmente em pacientes internados em hospitais de países em desenvolvimento, onde as taxas de mortalidade e os custos são extremamente elevados e significantes, principalmente quando cepas multirresistentes são implicadas ${ }^{18-20}$. Em um estudo realizado em uma UTI em Juiz de Fora - MG, em 2015, a taxa de mortalidade foi de 73,53\%, 25 óbitos dos 34 pacientes infectados por $\mathrm{KPC}^{20}$.

No estudo foram selecionados especificamente pacientes acima de 18 anos em que a média de idade destes foi de 53 anos, resultado similar de outros autores ${ }^{16-17,21}$. Em relação ao gênero mais acometido, teve predomínio para o sexo masculino em todas as regiões, com um total de 50\% das amostras, corroborando com outros estudos $^{17,22}$. Outros trabalhos apresentaram ausência de significância entre sexo/ idade e o desenvolvimento de infecções por microrganismos resistentes ${ }^{15}$, sendo uma das limitações encontradas para o levantamento de dados epidemiológicos para o estudo.

Em relação a K. pneumoniae resistente aos carbapenêmicos, a frequência nos hospitais brasileiros é alta, como relatado por outros autores ${ }^{21,23}$. Essas bactérias são mais frequentes em ambiente hospitalar do que outros coliformes, incluindo na superfície das mãos e de pisos e outros locais podem ser potencialmente um reservatório durante a ocorrência de surtos $^{24-25}$. Entre as cepas analisadas com fator KPC, a Klebsiella pneumoniae teve a maior porcentagem com $84 \%$ num total de todas as regiões, sendo a região Sul sua maior frequência com $92 \%$. No estudo de Dienstmann et al., (2010)8, também houve prevalência da mesma bactéria com um total de $70 \%$, assim como no estudo de Amorim et al, $(2014)^{26}$.

Desse modo, a detecção precoce de pacientes infectados ou colonizados pela KPC é de grande importância, uma vez que, esses microrganismos podem causar infecções graves e há escassez nas opções terapêuticas. Além disso, é ne-

Tabela 2: Prevalência dos antibióticos Betalactâmicos com maior resistência e sensibilidade em K. pneumoniae produtoras de KPC.

\begin{tabular}{|c|c|c|c|c|}
\hline Região Estado & Ano & Média da Maior Resistência (\%) & $\begin{array}{l}\text { Média de menor Re- } \\
\text { sistência (\%) }\end{array}$ & Média de Maior Sensibilidade (\%) \\
\hline \multicolumn{5}{|l|}{ Centro-Oeste } \\
\hline Goiás & $2006-2010$ & Ertapenem 100 & Gentamicina 57 & Fosfomicina 92,2 \\
\hline Mato Grosso & $2011-2013$ & Ertapenem 100 & - & - \\
\hline Espírito Santo & 2006 - 2010 & Ertapenem 100 & Gentamicina 31,15 & Fosfomicina 92,2 \\
\hline Minas Gerais & $2006-2013$ & Ertapenem 100 & Gentamicina 23,3 & Gentamicina 95,6 \\
\hline São Paulo & 2007 - 2009 & - & Gentamicina 12 & Amicacina 94,7 \\
\hline Rio de Janeiro & $2006-2015$ & Ertapenem 100 & Gentamicina 24,7 & Amicacina 95,7 \\
\hline $\begin{array}{l}\text { Rio Grande do Sul } \\
\text { Fonte: Autor (2020) }\end{array}$ & 2007 - 2014 & Ertapenem 97,2 & Gentamicina 28,2 & Fosfomicina 100 \\
\hline
\end{tabular}


cessário que sejam implementados protocolos de precauções de contato além de capacitações de higiene de mãos, proporcionando, assim, tratamento adequado dos pacientes e controle da disseminação desse tipo de mecanismo de resistência no Brasil e no mundo ${ }^{27-29}$.

Em países em desenvolvimento como o Brasil, a densidade de utilização de antibióticos é mais elevada, particularmente em unidade de cuidados intensivos ${ }^{30}$. Este alto consumo de antimicrobianos resulta na pressão seletiva de antibióticos, o principal responsável pela urgência de amostras resistentes e multirresistentes, que somando a existência de clones dominantes e a falha nas práticas básicas de prevenção e controle de infecções justificam a disseminação deste microrganismo no ambiente hospitalar ${ }^{6,31-34}$. Dados do Hospital Conceição - Uberlândia, evidenciam taxas muito elevadas de bactérias resistentes aos antibióticos ${ }^{35}$, particularmente relacionado com o alto consumo dos mesmos ${ }^{30,36-37}$.

A ausência de susceptibilidade ao Ertapenem já era esperada, considerando tratar-se de marcador de resistência aos carbapenêmicos, podendo estar relacionado diretamente à enzima KPC ou a outros mecanismos que diminuem a susceptibilidade a este antimicrobiano de forma específica $^{38}$. Nosso estudo, de revisão, mostrou $100 \%$ de resistência ao Ertapenem na maioria dos estados, sendo o PR, na região Sul, o único com uma porcentagem baixa de apenas $40 \%$ entre os anos 2009 e 2011.

A CIM relativa ao antimicrobiano Imipenem pode ser relacionada à diminuição de permeabilidade da membrana externa pela perda da porina, associada à produção de beta-lactamase ${ }^{38}$. Por outro lado, dados do MYSTIC ${ }^{39}$ apontam para um aumento na susceptibilidade de K. pneumoniae nos últimos anos, nos EUA, em função da prática de controle de disseminação de amostras resistentes. Diferente dos resultados encontrados em nosso estudo, em que o Meropenem foi o segundo antibiótico com maior resistência, sendo no estado do PR onde teve um

melhor resultado com apenas 32\% de resistência comparado aos demais.

$A$ ausência de susceptibilidade ao

Ertapenem já era esperada, considerando tratar-se de marcador de resistência aos carbapenêmicos, podendo estar relacionado diretamente à enzima KPC ou a outros mecanismos que diminuem a susceptibilidade a este antimicrobiano de forma específica

Assim como o estudo de ALMEIDA $(2013)^{40}$ estes resultados apresentaram a $\mathrm{K}$. pneumoniae como o microrganismo com maior resistência aos carbapenêmicos. Uma prevalência crescente destes microrganismos aumentou a dependência do uso de carbapenêmicos, antimicrobianos considerados como a opção para tratamento de infecçóes por bacilos Gram-negativos multirresistentes ${ }^{27}$. O fato crucial decorrente da emergência de amostras de K. pneumoniae com resistência aos carbapenêmicos nas poucas alternativas de tratamento, que incluem Colistina e Tigeciclina, que apresentam problemas em termos de toxicidade e eficácia, respectivamente ${ }^{27}$. Atualmente a escolha dos medicamentos é muito restrita, e o tratamento é realizado com a associação de três antibióticos: Polimixina B, Tigeciclina e Amicacina, ou em conjunto com os aminoglicosídeos.

Conforme estudo realizado em Porto Alegre $^{22}$, apresentou sensibilidade igual ou acima de $70 \%$ para a K. pneumoniae, a Gentamicina 70\%, a Tigeciclina 79.4\%, destacando-se a Amicacina com sensibilidade de $97,5 \%$, assim como o nosso estudo em que a Amicacina apresentou porcentagens maiores de $92 \%$ na região Centro-Oeste e Sudeste, sendo a Fosfomicina o destaque para a região Sul com $100 \%$ de sensibilidade para o estado do RS. Em outro estudo semelhante ${ }^{41}$ foram observados $100 \%$ de sensibilidade a gentamicina entre os isolados de $\mathrm{K}$. pneumoniae produtoras de KPC, assim como em nosso estudo em que o estado de MG apresentou resultado semelhante com $95,6 \%$ de sensibilidade. Lembrando que nenhum destes antimicrobianos tem relação adequada em infecções sistêmicas graves, é indicada, se possível, a associação de um ou mais antibióticos para o combate dessas infecções.

No estudo de Seibert et al. $(2014)^{23}$ apresentou $83 \%$ de resistência ao Meropenem e $91,5 \%$ ao Ertapenem, uma vez que o Ertapenem possuí um marcador de resistência aos carbapenêmicos, podendo estar relacionado diretamente à enzima $\mathrm{KPC}$ ou a outros mecanismos, que diminuem a sensibilidade a esse antimicrobiano de forma específica, como a produção de outras beta-lactamases e perda de porinas ${ }^{40,42}$. Em uma pesquisa a qual envolveu 62 isolados de K. pneumoniae produtoras de KPC, a resistência aos carbapenêmicos Imipenem, Meropenem e Ertapenem foi de $98 \%, 96 \%$ e $100 \%$, respectivamente ${ }^{43}$. Nesse estudo, os autores concluíram que a resistência ao Ertapenem representou o teste clínico mais sensível para a detecção 
da produção de KPC29,44-45. Sendo, todos estes resultados compatíveis com o do nosso estudo.

Há poucos estudos sobre a epidemiologia envolvendo os fatores de risco em pacientes infectados por K. pneumoniae no nosso país. Por outro lado, existem vários artigos publicados caracterizando a associação entre os fatores de risco e bactérias Gram-negativas produtoras de $\mathrm{KPC}^{46}$.

A Agência Nacional de Vigilância Sanitária (ANVISA) aprovou em 2018 o primeiro antibiótico específico para o combate de bactérias resistentes, incluindo a $\mathrm{KPC}^{47}$. O medicamento é uma combinação do antibiótico ceftazidima com o avibactam, sendo eficaz contra outras duas bactérias consideradas críticas para a saúde pública: a Pseudomonas aeruginosa e Enterobactérias produtoras de beta-lactamase de espectro ampliado ${ }^{48}$.

A KPC tem um alto potencial de disseminação sendo uma problemática de saúde global. A K. pneumoniae teve a maior porcentagem para bactérias produtoras de KPC, devido à sua localização plasmidial, a qual facilita a transferência do gene interespécies e tem sido motivo de preocupação em hospitais e instituições de saúde em todo o mundo ${ }^{23}$.

Em relação à multirresistência bacteriana, o uso indiscriminado de antimicrobianos é o principal responsável por tal problemática. A maior preocupação refere-se ao ambiente hospitalar e, principalmente, em pacientes gravemente enfermos e imunodeprimidos. Em contrapartida, outro fator contribuinte para a resistência é o descuido dos profissionais de saúde com o paciente gravemente colonizado ou infectado por microrganismo, tais como a não adesão e precaução de contato adequado e o não uso de EPI's.

\section{Há poucos estudos}

sobre a epidemiologia envolvendo os fatores

de risco em pacientes infectados por $K$. pneumoniae no nosso país. Por outro lado, existem vários artigos publicados caracterizando a associação entre os fatores de risco e bactérias Gramnegativas produtoras de KPC.

A região Sul, teve maior prevalência de isolados KPC mais especificamente no estado do Paraná, seguido da Região Centro-Oeste, destacando-se o Distrito Federal. Santa Catarina foi o estado mais preocupante em relação a grande taxa de isolados para $\mathrm{K}$. pneumoniae com fator $\mathrm{KPC}$, sendo essa uma bactéria multirresistente de grande preocupação mundial. Estados com maior taxa de desenvolvimento tem um acesso maior ao tratamento com antibióticos e o seu uso indiscriminado também é um fator preocupante para a disseminação de bactérias.

O Ertapenem mostrou-se o melhor indicador de resistência aos carbapenêmicos, podendo estar ou não relacionado à produção da enzima Klebsiella pneumoniae carbapenemase. Os aminoglicosídeos e a Tigeciclina apresentaram um bom percentual de sensibilidade, mostrando-se uma razoável opção terapêutica no tratamento de enterobactérias resistentes aos carbapenêmicos e sendo recomendada sua associação com um ou mais antimicrobianos ${ }^{23}$.

\section{CONCLUSÃO}

O KPC é um importante patógeno hospitalar e de crescente disseminação no mundo inteiro. A falta de medidas de prevenção pode ser o principal fator para a disseminação desses patógenos. Por conta disso, torna-se importante e necessária a rápida detecção laboratorial dos mecanismos de resistência desses microrganismos, assim como a adoção de medidas rigorosas e imediatas de prevenção e controle de disseminação, como a implementação de precauções de contato e tratamento adequado.

\section{REFERÊNCIAS}

1. Beirão EM, Furtado JJD, Girardello R, Ferreira FH, Gales AC. Clinical and Microbiological Characterization of KPC-producing Klebsiella pneumoniae Infections in Brazil. Braz J Infect Dis. 2011 [cited 2019 mar 4];15:69-73. Available from: https://doi.org/10.1590/ S1413-86702011000100013.

2. Tsakris A, Kristo I, Poulou A, Themeli-Digalaki K, Ikonomidis A, Petropoulou D, et al. Evaluation of Boronic Acid Disk Tests for Differentiating KPC-Possessing Klebsiella pneumoniae Iso- lates in the Clinical Laboratory. J Clin Microbiol. 2009[cited 2019 mar 4];47:362-367. Available from: https://pubmed.ncbi.nlm.nih. gov/19073868/

3. Hanes MS, Jude KM, Berger JM, Bonomo RA, Handel TM. Structural and biochemical characterization of the interaction between KPC-2 beta-lactamase and beta-lactamase inhibitor protein. Biochemistry. 2009 [cited 2019 mar 10];48:9185-9193. Available from: https://doi.org/10.1021/bi9007963 


\section{REFERÊNCIAS}

4. Kitchel B, Rasheed JK, Patel JB, Srinivasan A, Navon-Venezia S, Carmeli Y, et al. Molecular epidemiology of KPC-producing Klebsiella pneumoniae isolates in the United States: clonal expansion of multilocus sequence type 258. Antimicrob Agents Chemother. 2009[cited 2019 mar 10]; 53:3365-3370. Available from: https:// pubmed.ncbi.nlm.nih.gov/19506063/

5. Landman D, Bratu S, Quale J. Contribution of OmpK36 to carbapenem susceptibility in KPC-producing klebsiella pneumoniae. J Med Microbiol. 2009 [cited 2019 mar 11];58:1303-1308. Available from: https://pubmed.ncbi.nlm.nih.gov/19556371/

6. Queenam A; Bush K. Carbapenemases: the versatile -lactamases. J Clin Microbiol. 2007 [cited 2019 mar 11];20:440-458. Available from: https://cmr.asm.org/content/20/3/440

7. Bush K, Jacoby GA. Updated functional classification of beta-lactamases. Antimicrob Agents Chemother. 2010 [cited 2019 mar 11];;54:969-976. Available from: https://pubmed.ncbi.nlm. nih.gov/19995920/

8. Dienstmann R, Picoli SU, Meyer G, Schenkel T, Steyer J. Avaliação fenotípica da enzima Klebsiella pneumoniae carbapenemase (KPC) em Enterobacteriaceae de ambiente hospitalar. J Bras Patol Med Lab. 2010 [cited 2019 mar 13];46:23-27. Available from: https://dx.doi.org/10.1590/S1676-24442010000100005

9. Del Peloso PF, Barros MFL, Santos FA. Serratia marcescens KPC sepsis. J Bras Patol Med Lab. Out. 2010 [cited 2019 mar 13];46:365-367. Available from: https://doi.org/10.1590/S167624442010000500004.

10. Endimiani A, Perez F, Bajaksouzian S, Windau AR, Good CE, Choudhary Y, Hujer AM, Bethel CR, Bonomo RA, Jacobs MR. Evaluation of updated interpretative criteria for categorizing Klebsiella pneumoniae with reduced carbapenem susceptibility. J Clin Microbiol. 2010 [cited 2019 mar 16];48:4417-4425. Available from: https://pubmed.ncbi.nlm.nih.gov/20881179/

11. Fontana C, Favaro M, Sarmati L, Natoli S, Altieri A, Bossa MC, et al. Emergence of KPC-producing Klebsiella pneumoniae in Italy. BMC Rev. 2010 [cited 2019 mar 16];3:40. Available from: https:// doi.org/10.1590/S0100-40422006000400037

12. Silveira GP, Nome F, Gesser JC, Sá MM, Terenzi H. Estratégias utilizadas no combate a resistência bacteriana. Quim Nova. 2006 [cited 2019 mar 16];29:844-855. Available from: https://doi. org/10.1590/S1676-24442010000500004.

13. Van Boeckel TP, Gandra S, Ashok A, Caudron Q, Grenfell BT, Levin SA, et al. Global antibiotic consumption 2000 to 2010: an analysis of national pharmaceutical sales data. Rev Infect Dis. 2014 [cited 2019 mar 20];14:742-750. Available from: https:// pubmed.ncbi.nlm.nih.gov/25022435/

14. Brasil. Agência Nacional de Vigilância Sanitária. Plano Nacional para a Prevenção e o Controle da Resistência Microbiana nos Serviços de Saúde. Brasilia: ANVISA, 2017. Available from: http://portal.anvisa. gov.br/documents/33852/271855/Plano+Nacional+para+a+Preven $\%$ C $3 \% A 7 \%$ C $3 \% A 30+e+0+$ Controle+da+Resist $\%$ C3\%AAncia+Microbiana+nos+Servi\%C3\%A7os+de+Sa\%C3\%BAde/9d9f63f3-592b-4fe 1-8ff2-e035fccof31d. Acesso em: 16 Jun 2018.
15. Keynan Y, Rubinstein E. The changing face of Klebsiella pneumoniae infections in the community. Int J Ant imicrob Agents. 2007 [cited 2019 mar 20];30:385-389. Available from: https:// pubmed.ncbi.nlm.nih.gov/17716872/

16. Bergamasco MD, Barroso Barbosa M, de Oliveira Garcia D, Cipullo R, Moreira JC, Baia C, Barbosa V, et al. Infection with Klebsiella pneumoniae carbapenemase (KPC)-producing $\mathrm{K}$. pneumoniae in solid organ transplantation. Transpl Infect Dis. 2012 [cited 2019 mar 20];14:198-205. Available from: https://pubmed.ncbi.nlm.nih. gov/22093103/

17. Nordmann P; Girlich D; Poirel L. Detection of carbapenemase producers in Enterobacteriaceae by use of a novel screening medium. J Clin Microbiol. 2012 [cited 2019 mar 20];50:2761-6. Available from: https://pubmed.ncbi.nlm.nih.gov/22357501/

18. Hirsch EB, Tam VH. Detection and treatment options for pneumoniae carbapenemases (KPCs): an emerging cause of multidrug-resistant infection. J Antimicrob Chemother. 2010 [cited 2019 mar 25];65:1119-1125. Available from: https://pubmed. ncbi.nlm.nih.gov/20378670/

19. Falagas ME, Tansarli GS, Karageorgopoulos DE, Vardakas KZ. Deaths Attributable to Carbapenem-Resistant Enterobacteriaceae Infections. Emerg Infect Dis. 2014 [cited 2019 mar 25];20:1 -6. Available from: https://www.ncbi.nlm.nih.gov/pmc/articles/ PMC4073868/

20. Perna TDGS, Puiatti MA, Perna DH, Pereira NMM, Couri MG; Ferreira CMD. Prevalence of hospital infection with the bacteria klebsiella in an Intensive Care Unit. Rev Soc Bras Clin Med. 2015 [cited 2019 mar 25];13:119-123. Available from: http://files.bvs. br/upload/S/1679-1010/2015/v13n2/a4740.pdf

21. OLIVEIRA, A.O. et al. Epidemiologia da infecção hospitalar em Unidade de Terapia Intensiva. Rev Panam Infectol. 2009[cited 2019 mar 30];11:32-37.

22. Alves AP, Behar PR. Infecções hospitalares por enterobactérias produtoras de KPC em um hospital terciário do sul do Brasil. Rev AMRIGS. 2013 [cited 2019 mar 30];57:213-218. Available from: https:/pesquisa.bvsalud.org/portal/resource/pt/ biblio-998370

23. Seibert G, Hörner R, Meneghetti BH, Righi RA, Forno NLFD, Salla $A$. Infecções hospitalares por enterobactérias produtoras de Klebsiella pneumoniae carbapenemase em um hospital escola. Einstein. 2014 [cited 2019 mar 30];12:282-6. Available from: https://doi.org/10.1590/s1679-45082014ao3131

24. Cotrim ER, Rocha RDR. Klebsiella pneumoniae CARBAPENEMASE - KPC em Enterobacteriaceae: o desafio das bactérias multirresistentes. Pós em Revista. 2012 [cited 2019 abr 1];5.

25. Weterings V, Zhou K, Rossen JW, van Stenis D, Thewessen E, Kluytmans J, et al. An outbreak of colistin-resistant Klebsiella pneumoniae carbapenemase-producing Klebsiella pneumoniae in the Netherlands (July to December 2013), with inter-institutional spread. Eur J Clin Microbiol Infect Dis. 2015 [cited 2019 abr 1];34:1647-1655. Available from: https://pubmed.ncbi.nlm.nih. gov/26067658/ 


\section{artigo}

Marçal, T.V.G.; Costa, L.F.; Nicoletti, D.R.; Fernandes, M.T.C.; Amorin, B.; Hermes, D.

Incidência de KPC (Klebsiella Pneumoniae Carbapenemase) em adultos internados em hospitais nas regiões do Brasil de 2006 a 2016 : revisão bibliográfica

\section{REFERÊNCIAS}

26. Amorim RTCR, Oliveira KMG, Rodrigues SIC. Análise de aspectos epidemiológicos e clínicos e caracterização de genes de resistência das Enterobactérias produtoras de carbapenemases em um hospital do Distrito Federal. Brasília: Universidade de Brasília; 2014 [cited 2019 abr 1].

27. Hilal-Dandan R, Bruton LL. Goodman \& Gilman's: manual of phamacology and therapeutics. New York: McGraw-Hill, 2014 [cited 2019 abr 4]. Available from: https://accessmedicine.mhmedical.com/book.aspx?bookID=2189

28. Brunton LL, Chabner BA, Knollmann BC. As Bases Farmacológicas da Terapêutica de Goodman e Gilman. 12nd. Porto Alegre: AMGH, 2012 [cited 2019 abr 4].

29. Livermore DM. Fourten years in resistance. Int J Antimicrob Agents. 2012 [cited 2019 abr 4];39:283- 294. Available from: https://pubmed.ncbi.nlm.nih.gov/22386741/

30. Ambler RP. The structure of beta-lactamases. Philos Trans R Soc Lond B Biol Sci. 1980 [cited 2019 abr 4];289:321-331.

31. Moreira VC, Freire D. Klebsiella pneumoniae e sua resistência a antibióticos. Goiás: Universidade Católica de Goiás/IFAR;2011 [cited 2019 abr 6].

32. Davies J; Davies D. Origins and evolution of antibiotic resistance. Microbiol Mol Biol Rev. 2010 [cited 2019 abr 6];74:41733. Available from: https://www.ncbi.nlm.nih.gov/pmc/articles/ PMC2937522/

33. Ternent L, Dyson RJ, Krachler AM, Jabbari S. Bacterial fitness shapes the population dynamics of antibiotic-resistant and-susceptible bacteria in a model of combined antibiotic and anti-virulence treatment. J Theor Biol. 2015 [cited 2019 abr 20];71-11. Available from: https://pubmed.ncbi.nlm.nih.gov/25701634/

34. Iredell J, Tagg K, Brown J. Antibiotic resistance in Enterobacteriaceae: mechanisms and clinical implications. BM], 2016 [cited 2019 abr 20];6420. Available from: https://www.bmj.com/content/352/bmj.h6420

35. Sabino SS. et al. Incidência de infecção hospitalar em pacientes internados numa unidade de terapia intensiva clinica cirúrgica de um hospital universitário mineiro: a importância de microrganismos resistentes aos antimicrobianos. Am J Infect Control. 2016 [cited 2019 mai 2];5:95-96

36. Porto JP, Santos RO, Gontijo FPP, Ribas RM. Active surveillance to determine the impact of methicillin resistance on mortality in patients with bacteremia and influences of the use of antibiotics on the development of MRSA infection. Rev Soc Bras, Med Tropv. 2013 [cited 2019 mai 2];46:713- 718. Available from: http://dx. doi.org/10.1590/0037-8682-0199-2013.

37. Dantas RC, Ferreira ML, Gontijo-Filho PP, Ribas RM. Pseudomonas aeruginosa bacteraemia: independent risk factors for mortality and impact of resistance on outcome. J Med Microbiol, 2014 [cited 2019 mai 15];63:1679-1687. Available from: https:// pubmed.ncbi.nlm.nih.gov/25261066/

38. Woodford N, Eastaway AT, Ford M, Leanord A, Keane C, Quayle $\mathrm{RM}$, et al. Comparison of BD Phoenix, Vitek 2, and MicroScan Au- tomated Systems for Detection and Inference of Mechanisms Responsible for Carbapenem Resistance in Enterobacteriaceae. J Clin Microbiol, 2010 [cited 2019 mai 15];48:2999-3002. Available from: https://www.ncbi.nlm.nih.gov/pmc/articles/PMC2916632/

39. Rhomberg PR, Jones RN. Summary trends for the Meropenem Yearly Susceptibility Test Information Collection Program: a 10-year experience in the United States (1999-2008). Diagn micr infec dis, 2009 [cited 2019 mai 15];65:414-426. Available from: https://pubmed.ncbi.nlm.nih.gov/19833471/

40. Almeida VVP. Infecções por Klebsiella pneumoniae Resistente aos Carbapenemicos em Hospital de Nivel Terciário: Epidemiologia e Caracterização. Minas Gerais: Universidade Federal de Uberlândia; 2013 [cited 2019 mai 26]. Available from: https://repositorio. ufu.br/handle/123456789/12750

41. Soares VM. Emergência de Klebsiella pneumoniae produtora de carbapenemase (KPC) em um hospital terciário. J Bras Patol Med Lab. 2012 [cited 2019 mai 26];48:251-253. Available from: http://dx.doi.org/10.1590/S1676-24442012000400003.

42. Carvalho AT, Souza ES, Sousa DO, Costa MHA, Bahia GC, e Marsola LR. Higienização das mãos como estratégia para redução da incidência de infecções hospitalares em um hospital público. Rev Para Med, 2007 [cited 2019 mai 26];21: 80-80. Available from: http://scielo.iec.gov.br/scielo.php?script=sci_arttext\&pid=S0101-59072007000400018

43. Bratu S, Mooty M, Nichani S, Landman D, Gullans C, Pettinato $B$, et al. Emergence of KPC-possessing Klebsiella pneumoniae in Brooklyn, New York: epidemiology and recommendations for detection. Antimicrob Agents Chemothe, 2005 [cited 2019 mai 26];49:3018-20. Available from: https://aac.asm.org/content/49/7/3018

44. Yigit H, Queenan AM, Anderson GJ, Domenech-Sanchez A, Biddle JW, Steward CD, et al. Novel carbapenem-hydrolyzing beta-lactamase, KPC-1, from a carbapenem-resistant strain of Klebsiella pneumoniae. Antimicrob Agents Chemothe, 2008:45:1151-1161. Available from: https://pubmed.ncbi.nlm.nih.gov/11257029/

45. Andersson I, van Scheltinga $A C$, Valegård $K$. Towards new-lactam antibiotics. Cell Mol Life Sci, 2001 [cited 2019 jun 2];58:1897-1906. Available from: https://pubmed.ncbi.nlm.nih. gov/11766886/

46. Paterson DL, Bonomo RA. Extended-Spectrum -Lactamases: a Clinical Update. Clin Microbiol Rev, 2005 [cited 2019 jun 2];18:657-686. Available from: https://pubmed.ncbi.nlm.nih. gov/16223952/

47. ANVISA. Anvisa aprova novo antibiótico contra as superbactérias. Portal PubMed, 2018 [cited 2019 jun 2]. Available from: https://pebmed.com.br/anvisa-aprova-novo-antibiotico-contra-as-superbacterias/.

48. BRASIL. Agência Nacional de Vigilância Sanitária. Nota Técnica $N^{\circ} 01 / 2013$. Medidas de prevenção e controle de infecções por enterobactérias multirresistentes. Brasília: ANVISA, 2013 [cited 2019 jun 2]. Available from: http://www.saude.rs.gov.br/upload/1369161512_NOTA\%20TEC\%2001-2013\%20ANVISA.pdf. 